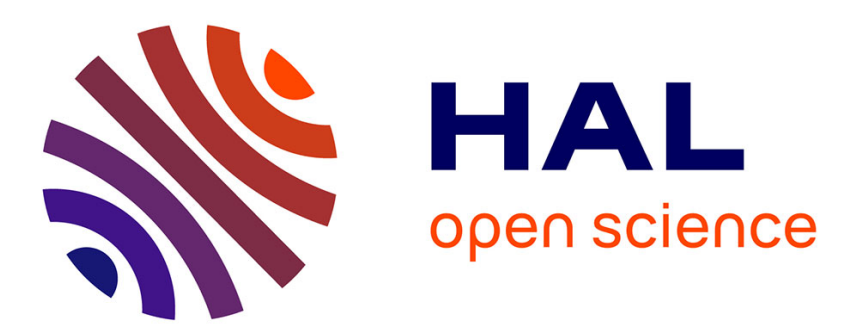

\title{
La perception de la famille et des liens chez les enfants confiés
}

Nathalie Chapon

\section{To cite this version:}

Nathalie Chapon. La perception de la famille et des liens chez les enfants confiés. Recherches familiales, 2018, 15 (1), pp.137-156. 10.3917/rf.015.0137 . halshs-01810265

\section{HAL Id: halshs-01810265 \\ https://shs.hal.science/halshs-01810265}

Submitted on 7 Jun 2018

HAL is a multi-disciplinary open access archive for the deposit and dissemination of scientific research documents, whether they are published or not. The documents may come from teaching and research institutions in France or abroad, or from public or private research centers.
L'archive ouverte pluridisciplinaire HAL, est destinée au dépôt et à la diffusion de documents scientifiques de niveau recherche, publiés ou non, émanant des établissements d'enseignement et de recherche français ou étrangers, des laboratoires publics ou privés. 
La perception de la famille chez les enfants confiés

Nathalie CHAPON

Cet article aborde la question spécifique et singulière de ce qui fait famille pour des enfants séparés de leurs parents et accueillis en famille d'accueil. Il questionne les dimensions familiales et parentales, les liens affectifs et électifs développés avec les parents, la famille d'accueil et les enfants présents au sein de cette famille. Quelle est la perception de la famille pour les enfants confiés? Qu'est-ce qu'une famille et qui en fait partie? Quels sont les sentiments exprimés vis-à-vis des parents et de la famille d'accueil ? Nous présenterons une partie des résultats d'une recherche réalisée sur ces questions auprès des enfants confiés, des parents mais aussi des familles d'accueil. Nous verrons que le tissage de liens et de "filiations " s'exprime différemment selon les situations de placement et les histoires de vie des enfants confiés et des familles. Etre séparé de ses parents et vivre au sein d'une famille dans un système de protection de l'enfance n'est pas aisé et confronte les enfants à bien des interrogations sur le "familial » et le "parental». Nous verrons que l'enfant confié vit une histoire familiale singulière composée de différents liens significatifs pour lui, de sentiments d'appartenance, qu'ils soient d'origine ou d'accueil ouvrant ainsi à une possible affiliation et à des configurations familiales bien identifiées.

\section{Introduction}

Les débats sur la famille dans la société française se sont cristallisés depuis quelques années sur les questions de reconnaissance possible à la fois socialement et juridiquement des différentes figures d' "attachement» (au sens large) circulant autour de l'enfant en familles recomposées, mais aussi en familles homoparentales. Ces questionnements n'ont pas épargné les familles d'accueil, en témoigne le nombre de colloques organisés actuellement en protection de l'enfance en France et en Europe qui abordent les questions d'attachement, d'engagement affectif et de filiation et qui tentent d'apporter des éléments de réponse à la fois dans le champ scientifique et dans celui de la pratique ${ }^{1}$.

En France on dénombre au 31 décembre 2015, 75000 enfants $^{2}$ confiés en famille d'accueil $^{3}$ par décision administrative ou judiciaire (hors placement direct par le juge). La famille d'accueil assume des tâches d'éducation et d'élevage, habituellement effectuées par la famille

1 The $9^{\text {th }}$ International Foster Care Research Network Conference, Continuity and disruption in foster care, 27-28 septembre, Université Paris-Nanterre, 2017 ; 5émes journées d'APFEL, Accueil familial en Europe: Sur le fil de l'histoire de vie, sur les fils de la parentalité plurielle, Le Gymnase, Lille, 1-2 décembre 2016 (voir la table ronde animée par Nathalie CHAPON, Accueil familial en Europe, parentalité d'accueil, parentalité plurielle, tour d'horizon européen); Nathalie CHAPON, «La parentalité d'accueil en Europe», Colloque Parentalité plurielle, APFEL, novembre, Université catholique Milan, 2015.

2 Direction de la recherche, des études, de l'évaluation et de la statistique (sous la direction d'Isabelle Leroux), L'aide et l'action sociale en France-Edition 2017, coll. Panorama, 2017, p. 98.

3 Dans une famille d'accueil, l'assistant familial est agréé pour accueillir de façon permanente, moyennant une rémunération, des mineurs et jeunes majeurs de moins de 21 ans à son domicile. Il exerce sa profession comme salarié au sein de sa famille désignée par l'expression «famille d'accueil». 
d'origine. Son rôle est de favoriser l'équilibre de l'enfant en lui offrant un contexte affectif, éducatif et matériel qui lui est indispensable.

Cette situation familiale particulière où un enfant est élevé par une autre famille sans pour autant avoir été abandonné par ses parents d'origine questionne les dimensions familiales et parentales ainsi que les liens affectifs et électifs développés; c'est-à-dire les liens filiatifs avec les parents mais également les liens électifs avec la famille d'accueil et les enfants présents dans cette famille ${ }^{4}$. Ce tissage de liens et de filiations s'exprime différemment selon les situations de placement et les histoires de vie des enfants confiés et des familles ${ }^{5}$.

Dans ce contexte particulier, il nous est apparu intéressant de mieux comprendre ce que signifie « la famille » pour les enfants confiés. Qu'est ce qui fait famille pour l'enfant? Qui fait partie de la famille de l'enfant? Il s'agit de préciser ce que les enfants entendent par la notion de famille. Quelle est leur perception de cette notion? De quelle famille parlent-ils ? Et quels sont leurs sentiments vis-à-vis de leurs parents mais aussi de la famille d'accueil ? Etre séparé de ses parents et vivre au sein d'une famille dans un système de protection de l'enfance n'est pas aisé et confronte les enfants à bien des interrogations ${ }^{6}$.

La famille d'accueil est une famille singulière qui, de fait, exerce une partie importante des fonctions parentales qui permettent à l'enfant d'établir des liens familiaux nouveaux et inédits, tout en étant enjointe à tenir une position professionnelle dans un dispositif de protection de l'enfant.

Notre approche conceptuelle s'inscrit dans le croisement de plusieurs apports théoriques. Nous inscrivons nos travaux dans la poursuite de ceux engagés par Paul Durning ${ }^{7}$ sur la suppléance familiale, avec lesquels nous croisons les approches d'Irène Théry ${ }^{8}$ et de Didier Houzel $^{9}$ sur la parenté, la parentalité ${ }^{10}$ et ses différentes composantes, ainsi que les travaux sur l'attachement multiple ${ }^{11}$, pour ainsi proposer une conceptualisation de la parentalité d'accueil et des modes de suppléance.

Nous ne développerons pas cette partie conceptuelle, nous préciserons seulement le concept de suppléance familiale, qui constitue le fait de suppléer les parents à un moment dans leur parcours parental, et celui de parentalité d'accueil, qui souligne les fonctions d'être parent et le fait de se vivre parent d'accueil en dehors du système de parenté. Cette problématique soustend l'ensemble de nos analyses.

4 Nathalie CHAPON, Parentalité d'accueil et relations affectives, Pup, 2014.

5 Françoise-Romaine OUELLETTE, Johanne CHARBONNEAU, Ercillia PALACIO-QUINTIN, Colette JOURDAN-IONESCU, Le placement en famille d'accueil : liens familiaux et dynamiques de réseau, INRS-Urbanisation, Culture et Société, 2001.

6 Vous retrouverez l'ensemble de cette recherche sur le site de l'Observatoire national de la protection de l'enfance: Nathalie CHAPON, Caroline SIFFREIN-BLANC, avec la collaboration de Gérard NEYRAND, La question des liens en accueil familial, qu'est-ce qui fait famille en famille d'accueil ?, Rapport de recherche ONPE, mars 2017. http://www.onpe.gouv.fr/appel-offre/familles-daccueilfamilles-dorigine-et-enfants-dans-laccueil-familial-appel-doffres

7 Paul DURNING, Education et suppléance familiale, CTNERHI, Paris, 1985.

8 Irène THERY, Anne-Marie LEROYER (dir.), Filiation, origines, parentalité. Le droit face aux nouvelles valeurs de responsabilité générationnelle, Odile Jacob, 2014.

9 Didier HOUZEL (dir.), Les enjeux de la parentalité, Erès, 1999.

10 Anne CADORET, «La parenté aujourd'hui : agencement de la filiation et de l'alliance », Sociétés Contemporaines, $\mathrm{n}^{\circ}$ 38, 2000 ; Agnès FINE, Claire NEIRINCK, Parents de sang et parents adoptifs, Droit et Société, LGD, 2000.

11 Observatoire national de l'enfance en danger/de la protection de l'enfance (dossier coordonné par Nathalie SAVARD), La théorie de l'attachement: une approche conceptuelle au service de la protection de l'enfance, Dossier thématique Oned, 2010. 


\section{Méthode de l'enquête}

\section{a. La Parole donnée aux enfants : aspects méthodologiques}

Donner la parole aux enfants dans le cadre d'un travail de recherche n'est pas toujours aisé. Les travaux français et québécois constatent que peu d'études explorent le point de vue de l'enfant pour comprendre leur univers quotidien ${ }^{12}$. Ils évoquent des difficultés méthodologiques et éthiques importantes. Dans la plupart des cas, une analyse de données secondaires est réalisée plutôt que de leur donner directement la parole ${ }^{13}$. La question de l'âge liée au développement cognitif de l'enfant se pose. L'enfant ou l'Infans est en effet, celui "qui ne parle pas ». Lorsqu'il sera ensuite capable de le faire, c'est le statut de cette parole qui sera remis en question. La parole énoncée de l'enfant, n'a pas socialement le même statut que la parole de l'adulte. Ainsi "l'enfant n'a d'abord pas la capacité de parler, il n'a ensuite pas de place pour le faire ${ }^{14}$, si bien que les chercheurs sont réticents à les interroger par crainte de les fragiliser davantage. Depuis une quinzaine d'années la recherche participative en sociologie de l'enfance encourage la prise en compte de la parole de l'enfant ${ }^{15}$, donnant ainsi à lire autrement le rapport dominant-dominé par l'accès « à la voix des acteurs faibles $\gg 16$.

Ce choix de rencontrer des enfants a demandé de prendre du temps pour obtenir les autorisations, puis avoir leur confiance. L'intérêt de la recherche était aussi de rencontrer l'ensemble des enfants qui gravitaient autour de l'enfant confié. Ainsi autour d'un seul enfant circule un nombre important d'autres enfants confiés ou non : que ce soit ses frères et sœurs, demi-frères et sœurs placés ou non, mais aussi les enfants vivant avec lui en famille d'accueil, l'ensemble des enfants confiés et les enfants de la famille d'accueil.

\section{b. Le choix de l'échantillon}

Deux conseils départementaux qui ont une politique de prise en charge et de suivi similaires ont été sollicités : Les Bouches-du-Rhône et le Vaucluse. Nous avons analysé 13 situations d'enfants dans les Bouches-du-Rhône et 12 situations dans le Vaucluse. Pour les deux départements, nous avons rencontré au total 71 enfants : 25 enfants sujets de l'étude, qui partagent leur quotidien avec 25 enfants confiés dans les familles d'accueil et 21 enfants de la famille d'accueil. Ce qui fait un total de 50 enfants confiés rencontrés, et leurs frères et sœurs d'accueil. Nous avons rencontré également 25 assistants familiaux et 10 parents (pour 10 situations). Soit un total de 106 entretiens pour l'ensemble de l'étude.

12 Carl LACHARITE, Catherine SELLENET, Claire CHAMBERLAND, La protection de l'enfance, la parole des enfants et des parents, Presses de l'université du Québec, 2015.

13 Louise HAMELIN-BRABANT, «La recherche auprès des enfants », Recherche et formation, $\mathrm{n}^{\circ}$ 52, pp. 77-89, Université de Laval, 2006.

14 COLLART Pierre, SOSSON Jehanne, La place de la parole de l'enfant, entre vérités et responsabilités, Bruylant-Academia, Belgique, 2007.

15 Adèle JONES, «Involving children and young people as researchers», in Sandy FRASER et al., Doing research with children and young people, Londres, Sage Publication, 2004 ; Régine SCELLES, Accueil des fratries: Analyse de l'expérience des professionnels travaillant dans le cadre de l'association SOS Villages d'Enfants. Rapport de recherche SOS Villages d'Enfants France, 2006 ; Régine SIROTA, «Entre enfants et parents la socialisation dans l'espace quotidien de la famille », $L a$ revue internationale de l'éducation familiale, 2015, $\mathrm{n}^{\circ} 37$.

16 Carl LACHARITE, Catherine SELLENET, Claire CHAMBERLAND, op.cit, p 1: « Le terme faible est emprunté à Michel de Certeau (1980), et celui d'acteur faible s'inscrit dans une volonté de réhabiliter les capacités et ressources d'action d'individus dominés, stigmatisés ». 
Cette démarche permet l'analyse de l'ensemble de la configuration familiale d'accueil : l'enfant sujet de l'étude, son assistant familial, le plus souvent une femme (un seul homme), les autres enfants confiés au domicile de la famille d'accueil, le nombre d'enfants pouvant aller jusqu'à 4 enfants confiés, puis les frères et sœurs de l'enfant sujet de l'étude (ceux-ci pouvaient être placés dans la même famille d'accueil, dans des familles d'accueil différentes, dans un établissement, ou encore être retournés ou être restés au sein de la famille d'origine) et enfin les parents d'origine. Dans le cadre de cet article nous présenterons une partie des résultats de la recherche en nous focalisant essentiellement sur le discours des 71 enfants.

Les enfants rencontrés dans le cadre de la recherche

\begin{tabular}{|c|c|c|c|c|}
\hline Dépt. & $\begin{array}{c}\text { Enfants } \\
\text { étude }\end{array}$ & $\begin{array}{c}\text { Autres enfants } \\
\text { confiés dans la } \\
\text { FA }\end{array}$ & $\begin{array}{c}\text { Enfants de la FA } \\
\text { présent au } \\
\text { domicile }\end{array}$ & Total \\
\hline 13 & 13 & 11 & 13 & 37 \\
\hline 84 & 12 & 14 & 8 & 34 \\
\hline Total & 25 & 25 & 21 & 71 \\
\hline
\end{tabular}

\section{c. Quelle est la démarche mise en œuvre dans le choix des situations ?}

Le choix des 25 situations a été fait afin de respecter la variété des situations des parcours de prise en charge des enfants à l'aide sociale à l'enfance.

Un protocole à partir de trois critères précis a été respecté tenant compte : de l'âge des enfants (6 ans à jeune majeur), de la durée des placements (courte, moyenne, longue), de la fréquence des rencontres avec les parents (régulières, irrégulières, pas de rencontre). Ces critères permettaient d'identifier différents types de suppléance que nous avions élaborés dans une précédente recherche ${ }^{17}$.

\section{Le traitement des données}

L'enquête repose sur un protocole qualitatif double : une analyse d'entretiens et des dossiers des enfants confiés.

Concernant le traitement des données, une triple grille d'analyse des différents entretiens a été mise en place pour chaque acteur rencontré au sujet de l'enfant. L'analyse sociologique croise des données qui mettent en relation les discours, à la fois des enfants, de leurs parents, de l'assistant familial, et des autres enfants, c'est-à-dire les enfants accueillis et tous ceux qui composent la fratrie. Une analyse thématique de type catégorielle a mis en évidence «des représentations sociales des interviewés à partir d'un examen de certains éléments constitutifs du discours $»^{18}$ sur la notion de famille, de ce qui constitue une famille, et de qui fait partie de la famille.

Dans un premier temps, nous avons réfléchi à la notion de famille, de ce qui pouvait constituer une famille pour l'enfant. Des éléments significatifs donnent une définition commune de l'entité familiale pour les enfants.

Dans un second temps, nous avons voulu comprendre l'intensité des sentiments des enfants envers leurs parents et leur famille d'accueil. Un outil d'analyse spécifique a été utilisé en demandant à l'enfant d'exprimer son ressenti par rapport aux différents membres de sa famille

17 Nathalie CHAPON, Relations affectives et parentalité, Pup, Aix-en-Provence, 2014.

18 Raymond QUIVY, Luc VAN CAMPENHOUDT, Manuel de recherche en sciences sociales, Dunod, 1998. 
d'accueil et d'origine. Bien que reconnaissant l'importance des outils permettant de mesurer l'attachement de l'enfant aux différentes figures d'attachement significatives pour lui ${ }^{19}$, l'intérêt ici n'était pas de mesurer l'attachement de l'enfant à ses proches mais simplement de souligner l'intensité de ses sentiments. Dans ce sens nous avons utilisé un outil spécifique pour les enfants, simple d'utilisation et de compréhension à l'aide de chiffres et d'émoticônes figurant un visage associé à une émotion, un ressenti. L'émoticône apporte une information sur l'émotion, le sentiment de l'auteur dans un contexte précis.

Le croisement de l'ensemble de ces données avec des facteurs socio-démographiques a permis d'affiner différentes définitions de la famille abordées par les enfants, et de proposer quatre configurations familiales.

Une analyse sociologique des dossiers des enfants confiés à partir de repères historiques et familiaux dans leur parcours de prise en charge est venue éclairer et compléter les propos des enfants.

\section{Résultats de l'enquête}

\section{a. Qu'est-ce qu'une famille pour l'enfant ?}

Cette question «Qu'est-ce qu'une famille? » posée aux enfants montre que la notion de famille peut être appréhendée de différentes façons pour les personnes confrontées à une situation de placement en famille d'accueil. Comprendre ce que la famille représente pour les principaux intéressés s'avère une source de connaissance appréciable, tant au niveau de ce que les enfants entendent par la notion de famille, que pour appréhender les différentes configurations familiales vécues.

\section{Une famille, c'est être ensemble}

Lorsqu'on demande aux enfants de définir la famille, ils en donnent une définition simple à partir du regroupement d'individus spécifiques, et l'expression de valeurs générales. Tout d'abord pour qu'une famille existe pour les enfants, il y a forcément des parents, et des enfants qui vivent ensemble sous le même toit. Lorsque les membres de la parenté sont convoqués, ils le sont le plus souvent dans le sens d'une désignation générale de fonction parentale et non d'une filiation déterminée par les ascendants et les descendants. Le noyau familial identifié fait l'objet de ce que Moscovici ${ }^{20}$ nomme «l'altruisme participatif », c'est-àdire la désignation d'une communauté d'individus à laquelle on s'identifie. "Moi ma famille, $c$ 'est Richard, Martine et Carole ».

Pour Lila âgée de 8 ans "Ce n'est pas tout à fait pareil, ma famille, pour moi c'est ma famille d'accueil, ma mère de cour, elle m'a élevée, ma maman et mon papa je ne les ai pas beaucoup vus, donc c'est plus ma famille d'accueil, c'est eux qui m'ont éduquée, élevée - c'est comme ça être en famille d'accueil, ma vrai mère elle a des petits soucis, je crois qu'elle s'appelle Chantal ma vraie mère, je ne sais pas - pour moi, c'est ma famille d'accueil ».

Pour Cyndie, "une famille c'est être tous ensemble ». Cyndie considère avoir une famille composée de deux entités. Celle d'accueil et celle d'origine avec laquelle elle compose, ses frères et sœurs placés qu'elle voit, ses parents d'origine. Leur famille est une mosaïque composée de deux versants, l'un d'accueil, l'autre d'origine, qui s'imbriquent en fonction de l'histoire du placement.

19 Nicole GUEDENEY, Antoine GUEDENEY, L'attachement, concepts et applications, Masson, 2002.

20 Serge MOSCOVICI, Psychologie sociale des relations à autrui, Nathan, 1994. 


\section{La famille, c'est s'aimer}

Au-delà du simple regroupement d'individus vivant ensemble, des valeurs communes animent le groupe et des sentiments affectifs forts. S'aimer apparaît encore plus important que de vivre ensemble. "La famille... c'est-à-dire, pour moi je ne sais pas trop comment expliquer... ben... d'être ensemble, de s'aimer, avoir de l'amour entre nous ». L'amour est à chaque fois l'élément structurant la famille, le ciment faisant lien entre ses membres. Selon Edouard Shorter ${ }^{21}$ "le ciment affectif de la famille englobe plus que le mari et la femme; il maintient aussi leurs enfants à l'intérieur de cette unité sentimentale ». Dans la définition donnée par les enfants, la filiation est le plus souvent occultée au profit des sentiments d'affection exprimés entre l'ensemble des personnes partageant le même toit.

Laurent est un enfant qui a été placé à l'âge de 6 ans dans sa famille d'accueil. Il a aujourd'hui 16 ans. Il a toujours maintenu des relations avec son père une fois par mois ; en revanche il n'a plus de nouvelles de sa mère depuis son placement. Pour lui la famille est d'abord aimante, et fidèle, c'est-à-dire présente quels que soient les évènements de la vie. " Pour moi une famille c'est des gens qui t'aiment, en qui t'as confiance, qui sont fidèles et voilà ! Ma famille, c'est la famille à mamie (son assistante familiale) et voilà ! ... Mon père, mouais... mais pas ma mère! ». Puis il parle de Mamie, en disant qu'elle est très importante pour lui «Elle me dit: tu es mon enfant! Elle me le dit... je t'aime des fois! Moi je le dis pas, non! Je ne lui dis pas mais elle le sait!».

\section{Une famille, une présence}

Une famille c'est être ensemble, s'aimer et être présent. Laurent considère la famille de l'assistante familiale comme sa propre famille. Il exprime beaucoup d'amour pour elle et cela semble réciproque. Il se sent bien dans cette famille, il se sent chez lui. Il ne voit pas sa mère depuis ses 7 ans, et vit son absence depuis plus de 10 ans comme un abandon. De même, il n'a aucun contact avec ses demi-frères et sœurs, et ne souhaite pas en avoir, car il ne les considère pas comme sa famille. En revanche, il voit son père de temps en temps, mange avec lui, mais ne veut pas dormir chez lui car cela pourrait le stresser. Pour lui dans une famille, on est ensemble, on est présent, on n'abandonne pas un de ses membres.

"Ma mère, la dernière fois que je l'ai vue, j'avais 7 ans! Donc pour moi ce n'est pas un membre de ma famille! Non parce qu'un membre de la famille on ne l'abandonne pas! C'est la logique même! Ouais pas ma mère!».

La notion de famille est rattachée pour les enfants à leur quotidien, aux échanges, à la présence de sentiments affectifs, à des liens tissés dans un cadre familial.

\section{b. Que disent les enfants de leurs sentiments vis-à-vis de leur famille d'accueil et de leurs parents}

Vinciane, assistante familiale relate un échange avec l'enfant qu'elle garde depuis plus de 10 ans.

"Souvent il me demande: 'Comment je l'aime? Comment tu m'aimes ?' Je lui réponds: 'Comment je t'aime? Mais je t'aime profondément, oui je t'aime profondément'” ».

D'après les résultats de notre recherche sur l'ensemble des 25 enfants confiés, la quasi-totalité des enfants expriment un investissement affectif fort à l'égard de leur famille d'accueil. Ils

21 Edouard SHORTER, Naissance de la famille moderne, Seuil, 1977. 
nomment en priorité la famille d'accueil quand ils abordent la question de la famille, puis leur fratrie ; leurs parents arrivent le plus souvent en dernière position. L'intensité des sentiments exprimés est souvent plus forte pour la famille d'accueil et ses membres que pour la famille d'origine. Les $2 / 3$ des enfants estiment avoir des sentiments très forts pour leur famille d'accueil, avec une expression au maximum. On constate donc un décalage affectif important entre les deux entités familiales, la famille d'accueil étant la structure familiale la plus investie par les enfants confiés.

L'expression des sentiments de l'enfant à l'égard de ses parents apparait partagée et nuancée $^{22}$. Les enfants opèrent une distinction affective entre le père et la mère. Ils expriment des sentiments plus modérés à l'égard de leur père que pour leur mère ${ }^{23}$.

Nous verrons que l'analyse des configurations familiales pour l'enfant vient confirmer ces éléments. Un placement en bas âge, qui s'inscrit dans la durée, des parents plus ou moins absents, avec des visites très irrégulières voire inexistantes sur plusieurs mois, une famille d'accueil qui écoute, soutient l'enfant, qui l'éduque au quotidien, qui l'investit aussi affectivement, ce sont autant d'éléments apportant du sens aux nuances affectives observées.

\section{c. L'éloignement des parents d'origine, la présence au quotidien de la famille d'accueil}

Dans des situations de déperdition de liens, l'enfant placé, à son tour, éconduit souvent le parent absent depuis de nombreuses années, considérant qu'il ne fait pas ou plus partie de sa famille ; c'est ce que nous avons pu constater au sujet de la mère des enfants rencontrés, identifiée par ces enfants comme le plus souvent absente. Les propos sont empreints d'émotion, parfois de colère et de ressenti.

\section{Une méconnaissance du parent, un ressenti face à la mère}

Un certain nombre d'enfants confiés ne connaissent pas vraiment leurs deux parents. Or, pour les enfants on ne peut nommer une personne que l'on ne connait pas, comme faisant partie de ses proches et de sa famille. On comprend que pour l'enfant qui répond "Ma mère, je ne la connais pas », la relation à la mère apparait problématique. Quasiment la moitié des enfants ont un discours marqué par l'incompréhension, l'opposition à l'égard de leur mère lorsqu'elle interrompt les visites, coupe les contacts avec eux et s'absente pendant de nombreuses années (11 enfants). Ils ne s'expliquent pas son attitude de retrait, son manque d'investissement affectif. Plusieurs années après, lorsqu'ils évoquent les évènements du moment de la rupture, ils ne comprennent pas son attitude, estiment ne pas connaitre leur mère, et apparaissent marqués par cette méconnaissance qu'ils identifient comme de l'indifférence de sa part.

Nina est une enfant de 10 ans au moment de l'enquête, confiée à une famille d'accueil depuis sa naissance. Sa mère l'a abandonnée à la naissance, seul son père a maintenu les visites jusqu'à son décès lorsque Nina avait 7 ans. Les expressions employées par Nina pour parler de sa mère reflètent une mise à distance. Elle emploie l'expression «celle qui m'a mis au monde », et considère sa mère comme une étrangère. Les mots de Nina pour parler de sa mère sont ceux du registre du singulier, de l'étranger, "cette personne, c'est une dame, je ne sais

22 - 6 enfants seulement estiment avoir des sentiments très forts pour leur(s) parent(s), et dans le même temps 5 enfants expriment un investissement affectif très faible à leur égard.

23 L'expression des sentiments à l'égard de la mère se situe aux deux extrémités de l'échelle des sentiments (2-10) alors que pour le père les sentiments sont plus nuancés autour de 4 à 7 , avec un discours des enfants qui semble moins radical. 
rien d'elle ». Les mots pour la mère sont ceux de la méconnaissance : «Je ne la connais pas ». Dans ce cadre elle ne peut appartenir au groupe familial identifié par l'enfant.

D'où l'évidence pour les enfants de ne pas nommer cette personne comme un membre de leur famille. Un étranger est une personne que l'on ne connait pas, il ne fait donc pas partie de la famille. Même si cette femme a mis au monde cet enfant, elle demeure à l'extérieur du groupe familial pour l'enfant. En cela, ces constats rejoignent les analyses socio-anthropologiques : le géniteur n'est pas obligatoirement identifié comme un parent. Pour Nina, sa mère ne peut pas faire partie de sa famille. "Ma mère elle n'est pas du tout dans ma famille, largement pas. J'éprouve aucun sentiment... je n'ai pas de sentiment, je ne peux pas dire si je la déteste parce que je ne la connais pas... mais je ne peux pas dire que je l'aime car je ne la connais pas! Elle m'a quand même abandonnée ». Cette histoire de rupture de lien n'est pas isolée car elle constitue la moitié des situations étudiées.

C'est le cas de Cylia et de l'ensemble de sa fratrie, qui depuis 10 années n'ont pas vu Nadine, leur mère, qui souhaite aujourd'hui de nouveau rencontrer ses enfants; mais c'est aussi l'histoire de Mélodie, âgée de 25 ans, qui ne voit plus sa mère depuis de nombreuses années, ou encore Lilian et Bruno, qui ne connaissent pas leurs parents. Il semble alors tout à fait normal pour les enfants ne pas identifier cette mère inconnue comme un membre de leur famille.

\section{Pourtant des pères plus souvent absents que les mères}

Qu'en est-il de la présence des pères et du rythme des rencontres paternelles pour les enfants confiés ? La mère serait-elle plus souvent absente auprès de l'enfant que le père ? Ou bien s'agit-il d'une représentation de l'enfant face à un manque maternel plus largement ressenti, le père étant lui aussi également absent auprès de l'enfant ? Cela correspond-il à des faits objectivables comme le rythme des visites entre les parents et l'enfant?

Sur les 25 enfants sujets de l'étude, 11 n'ont plus de relation avec leur mère, et 15 n'ont plus de relation avec leur père. Plus le placement de l'enfant dure dans le temps, plus le risque est grand de vivre une déperdition des liens avec un des deux parents. Les enfants les plus touchés par cette déperdition sont ceux entrant dans la pré-adolescence et l'adolescence, les plus petits apparaissent moins touchés. Si ces chiffres confirment une absence plus fréquente du père, et des pré-ados plus souvent concernés que les autres, cela semble rendre compte du statut privilégié de la relation à la mère, dont la perturbation serait plus durement vécue que celle au père, en conformité aux représentations et aux discours dominants. Ainsi l'arrêt des visites et des contacts entre les enfants et leur mère pendant plusieurs années, au moment de la petite enfance jusqu'à l'adolescence, a provoqué une réelle rupture du lien maternel, alimentée par l'incompréhension des enfants, une souffrance silencieuse, et un attachement indéfectible à la famille d'accueil, présente tout au long de ces années. Cette perte d'un lien affectif primordial entraîne avec le placement la création d'un autre lien nécessaire à l'équilibre, soit avec l'assistante familiale et sa famille, soit avec une autre figure familiale, l'enfant comme le jeune manifestant un irrésistible besoin de se sentir lié, aimé et considéré comme faisant partie d'une famille. On retrouve ici la notion de pulsion d'attachement ${ }^{24}$, qui serait nécessaire à l'édification de l'individu, ou du sujet. La famille serait le lieu où cet attachement est possible. La déperdition des relations affectives avec l'un des parents, ou les deux parents conduisent lentement l'enfant à se reconstruire en dehors de sa structure familiale d'origine et à considérer que sa structure familiale de référence est celle de sa famille d'accueil.

24 Bernard GOLSE, «Entre psychanalyse et attachement, le concept de pulsion d'attachement comme moyen de penser la symbolisation en absence et en présence de l'objet », Dialogue, n 175, 2007. 


\section{d. La question de la nomination}

\section{Etre mère et/ou être maman}

Comment dire le lien ? Les mots sont nombreux dans le registre des sentiments : amour, tendresse, ou affection. Si le premier est associé au couple, le second renvoie à « la proximité physique des gestes maternels »; quant au troisième, il est généralement utilisé pour désigner l'attachement entre des personnes de générations différentes ${ }^{25}$. Les enfants sont davantage spontanés que les adultes dans leur expression et ont souvent des mots directs pour dire les choses. Quand on leur demande de nous expliquer comment ils aiment, les mots sont simples, alors que l'adulte hésite, tant il y voit le plus souvent le lieu d'une grande complexité. Un enfant fait, par exemple, une distinction entre la mère, celle qui l'a mise au monde, et la maman celle qui s'occupe de lui, c'est-à-dire dans le cadre de l'accueil familial, l'assistante familiale : "Ma mère, je préfère l'appeler ma mère, celle qui m'a mise au monde, quand on va aux visites, elle ne nous parle presque pas, ma maman c'est Sylvie. »

Un autre enfant, plus âgé, Christophe, 16 ans, procède plutôt par analogie : "Nathalia c'est comme une mère, je suis arrivée j'avais 14 mois, elle m'a tout appris, comme faire les lacets, c'est comme une mère, elle a fait le travail qu'une mère fait. »Christophe évoque ainsi un amour de type maternel pour son assistante familiale, qui s'est occupée de lui, et un désintérêt par rapport à sa mère biologique considérée comme une étrangère : "C'est comme si c'était ma mère, c'est un amour maternel. Par rapport à ma mère c'est différent, je ne la vois plus depuis des années...il n'y a pas longtemps elle voulait nous revoir, elle a essayé de revenir dans ma vie, c'est une personne qui m'apporte rien du tout. L'éducatrice lui a dit qu'on ne voulait plus la voir. »

Cette analyse rejoint celle déjà réalisée dans nos précédents travaux ${ }^{26}$, et confirme la distinction déjà repérée entre les différentes façons de nommer les acteurs parentaux. Derrière ces différentes façons d'utiliser la nomination, on retrouve la distinction faite par les enfants entre les rôles et les statuts, notamment en ce qui concerne la position maternelle, voire parentale.

\section{La Nomination, un faux problème?}

Compte tenu de la conscience qu'ont les enfants des différentes places tenues par les figures familiales qui les entourent, on peut se demander si la focalisation des travailleurs sociaux sur la nomination de type parental (maman, papa) par l'enfant pour sa famille d'accueil n'est pas un faux problème, ou du moins n'est pas révélatrice d'au moins deux choses: leur attachement à une conception traditionnelle de la filiation qui ne permet d'identifier et de nommer comme parents d'autres figures parentales que les parents d'origine, puis le caractère contradictoire des injonctions auxquelles en tant que professionnels sont confrontés les assistants familiaux : faire «comme si » c'était notre enfant, «comme si » c'était notre parent, sans pouvoir le nommer...

La plupart des enfants ont une vision juste de la situation vécue en famille d'accueil, ils ont une connaissance des différentes places de chacun dans les structures familiales, ils distinguent fortement leurs parents et leur famille d'accueil et manifestent une absence de confusion des rôles. L'importance de l'amour familial vécu et l'immersion de leur quotidien

25 Claudine ATTIAS-DONFUT, Nicole LAPIERE, Martine SEGALEN, Le nouvel esprit de famille, Odile Jacob, 2002.

26 Nathalie CHAPON, Gérard NEYRAND, «Parentalité et famille d'accueil », Dialogue, $\mathrm{n}^{\circ} 167$, 2005.

Nathalie CHAPON, 2014, op.cit. 
dans une relation affective d'accueil parfois très forte expliquent l'emploi de mots, la désignation de l'autre comme faisant partie de la famille, la désignation de l'autre en référence à la parenté. La nomination ne signifie ni confusion, ni usurpation. Quasiment tous les enfants rencontrés connaissent leurs parents, qu'ils les rencontrent ou pas. Il est vrai que l'absence de contact parental, la recherche d'un foyer sécurisant, le besoin de chaleur affective, un placement précoce et de longue durée, la perte d'une figure parentale sont autant d'éléments favorables pour glisser vers une nomination parentale qui est basée sur l'exercice d'une fonction, et non la révélation d'un statut. La confusion demeure ici entre statut, rôle et l'exercice des fonctions parentales. Les parents conservent leur statut mais sont le plus souvent dépourvus de l'ensemble des fonctions parentales, si ce n'est lors des visites - elles sont alors encadrées - ou des hébergements en week-ends et vacances scolaires, quand les fonctions parentales sont exercées en discontinuité et évaluées par les services. Ainsi, Cyndie âgée de 15 ans, accueillie depuis l'âge de deux ans dans la famille d'accueil, appelle Martine et Richard, «maman» et «papa», alors qu'elle rencontre son père 1 fois par mois et de nouveau sa mère 1 fois tous les deux mois après 9 ans d'absence. La nomination de Martine et Richard papa et maman, n'enlève rien aux statuts parentaux, elle n'est que le reflet d'un attachement singulier qui fait sens pour l'enfant et sa famille d'accueil.

\section{L'assistante familiale : ma maman}

Nina explique qu'elle aime son assistante familiale «comme si » elle était sa vraie maman, puisqu'elle ne connait pas sa mère. Pour elle, sa mère est une personne étrangère (sentiment identique chez Cyndie, Khélia, Christophe), une dame dont elle ne sait rien. Elle ne peut ni l'aimer, ni la détester puisqu'elle ne la connait pas. Les mots des enfants sont des mots qui identifient une réalité comme étant la leur, empruntant les désignations communes dans le champ de la parentalité pour nommer ces acteurs parentaux qui sont «comme » une mère ou « comme » un père. Ils indiquent ainsi à la fois la connaissance de la définition sociale de ces termes et la conscience de ce que leur situation a d'atypique. Ils pointent ainsi la dissociation entre la composante socio-juridique de la parenté, associée au biologique, et la composante plus relationnelle et affective des situations familiales, renvoyant au registre des affiliations sur lequel insiste la parentalité. La nomination vient alors insister sur ce clivage, soit en opposant mère (biologique) et maman (affective), soit en indiquant la différence de registre par le «comme », en rappelant la place prise par ces figures substitutives vécues comme les «vraies » figures de la parentalité. "Mamoure, je l'aime comme si c'était ma vraie maman, pour moi je la prends comme ma mère. Ma mère je la connais pas, c'est une dame, je ne sais rien d'elle, je ne sais pas à quoi elle ressemble ; si elle voulait me reprendre, je dirai non. »

Cette analyse vient interpeller avec force le positionnement institutionnel, confondant désignation et nomination, ainsi que statut et rôle parentaux. Certes les fonctions parentales sont dissociées, les lieux de vie aussi, mais les statuts sont posés par les cadres biologiques juridiques, et bien que les rôles parentaux soient différenciés, parcellarisés, les places occupées par les différentes figures parentales ne sont pas opposables, ni «confondables ». Il ne peut dans ce sens y avoir de confusion de statut, chacun connaissant la place de l'autre. Reconnaître ce qui se joue affectivement dans la famille d'accueil éviterait bien des souffrances pour l'ensemble des acteurs.

\section{Quatre configurations familiales pour les enfants}

Nous avons vu que la perception de la notion de famille pour les enfants confiés rejoint l'analyse de leurs ressentis vis-à-vis de leur parent et leur famille d'accueil. Ainsi les sentiments affectifs exprimés sont majoritairement les plus forts pour la famille d'accueil, 
alors que ceux exprimés pour les parents sont bien plus complexes et négatifs. Les ruptures de lien, des difficultés relationnelles avec les parents, l'implication affective de la famille d'accueil, explicitent ces distinctions. Face à ces situations, les ruptures avec la mère sont davantage soulignées par les enfants et vécues difficilement. L'investissement auprès de l'assistante familiale et de sa famille est d'autant plus grand, mais il n'y a aucune confusion pour les enfants au sujet des places de chacun. Cette première analyse nous conduit à poursuivre de façon plus précise la définition de la famille pour les enfants. S'agit-il d'un enfant «partagé entre deux familles »? Ou bien fait-il un choix précis entre ses deux entités familiales ? C'est ce que nous allons voir avec les configurations familiales. L'enquête dégage quatre formes de famille pour les enfants confiés.

\section{Quatre configurations familiales}

Les enfants ont une représentation de la famille qui s'oriente vers quatre possibilités : d'un côté, leur famille d'origine, celle de leur naissance, et de l'autre côté, leur famille d'accueil, celle de l'éducation et du quotidien. Ces distinctions s'opèrent selon un subtil mélange des enfants, opérant un ajustement selon leur histoire entre ce qu'ils nomment "celle d'ici, là où je vis » la famille d'accueil, et « celle de temps en temps » la famille d'origine.

La construction de ces références familiales se fait en fonction du temps passé dans le lieu de vie, secondairement de la structure de la famille. La famille d'accueil d'un côté, les parents d'origine de l'autre, associés à la notion de partage du temps, pour l'une le quotidien, avec une majorité du temps, et pour l'autre un temps parcellaire, ponctué par les visites à domicile ou en institution. Le critère caractéristique de ce qui fait famille est donc le temps de coprésence, venant rendre compte des raisons de la prégnance de l'affect dans les situations étudiées. Le positionnement familial des enfants entre les quatre configurations est très clair et ne prête pas à confusion, soit l'enfant ne peut choisir entre ses deux familles, soit il fait un choix. Dans la majorité des cas (15/24), un choix est fait par l'enfant.

Quatre configurations se dessinent :

- soit ils considèrent comme secondaire leur famille d'origine et donnent à leur famille d'accueil la première place en termes de référence familiale, du fait de l'éducation reçue, des soins apportés et des sentiments éprouvés : «Ma famille, celle où je vis » (10/24) ;

- soit, ils mettent en parallèle de manière identique les deux familles en faisant référence dans un cas à leur naissance, et dans l'autre à leur éducation quotidienne : «Mes deux familles » (9/24);

- soit, l'enfant considère ses parents comme leur seule référence familiale : «Mes parents seulement $\gg(1 / 24)$;

- soit ils considèrent ne pas vraiment avoir de famille et ne font référence ni à l'une, ni à l'autre : "Ni mes parents, ni ma famille d'accueil » (4/24).

Les deux configurations les plus représentées renvoient à la catégorie "Ma famille, celle où je vis », où l'enfant considère sa famille d'accueil comme sa famille de référence et «Mes deux familles », où l'enfant considère avoir deux familles et ne peut choisir l'une d'elle.

La première catégorie "Ma famille, celle où je vis », concerne 10 enfants. L'enfant met en avant l'importance de ce qu'il vit dans sa famille d'accueil, des apprentissages, du soutien. Leur famille "c'est ici, celle où je suis! parce qu'ils m'ont appris à faire plein de choses, c'est eux qui m'ont accueillie! ». Pour la catégorie «Mes deux familles » qui concerne 9 enfants sur 24. L'enfant a deux familles, et dit les aimer de façon identique. 
Les deux dernières catégories «Ni mes parents, ni ma famille d'accueil » et «Mes parents seulement », sont minoritaires, et ne concernent pour chacune d'elle que peu d'enfants. La catégorie «Ni mes parents, ni ma famille d'accueil » interpelle, car même s'il s'agit pour la plupart de placements tardifs, malgré l'accueil familial apporté le jeune ne retrouve pas de références familiales solides, il va les chercher à l'extérieur du cadre de référence d'origine et d'accueil.

Nous allons présenter chaque configuration à partir de cas cliniques et de schémas représentant la perception familiale de l'enfant. Les chiffres représentent l'investissement affectif exprimé par l'enfant.

\section{a «Ma famille, celle où je vis »}

Dans la majorité des cas, les enfants se positionnent dans la catégorie «Ma famille, celle où je vis », estimant comme une renaissance le fait d'avoir été placés dans leur famille d'accueil. Ils vivent l'évènement du placement comme une chance. Certains enfants, en rupture relationnelle parentale depuis plusieurs mois, voire plusieurs années, ont même tendance parfois à tenter d'oublier leur famille biologique tant ils se sentent faire famille avec leur famille d'accueil. Certains aimeraient être adoptés par la famille d'accueil, tout en gardant de temps en temps des contacts avec leurs parents. Cette adoption leur apporterait la sécurité de savoir qu'ils resteront jusqu'à leur majorité dans la famille d'accueil, et qu'ils ne vivront plus dans l'inquiétude d'un probable retour. Nous pensons à Chris, qui voit son père régulièrement mais qui ne souhaite absolument pas retourner vivre chez lui. Celui-ci lui dit souvent qu'il souhaite la récupérer, alors que ses conditions de vie sont peu propices à cela. Chris vit ces demandes comme des menaces, et s'en trouve très inquiétée.
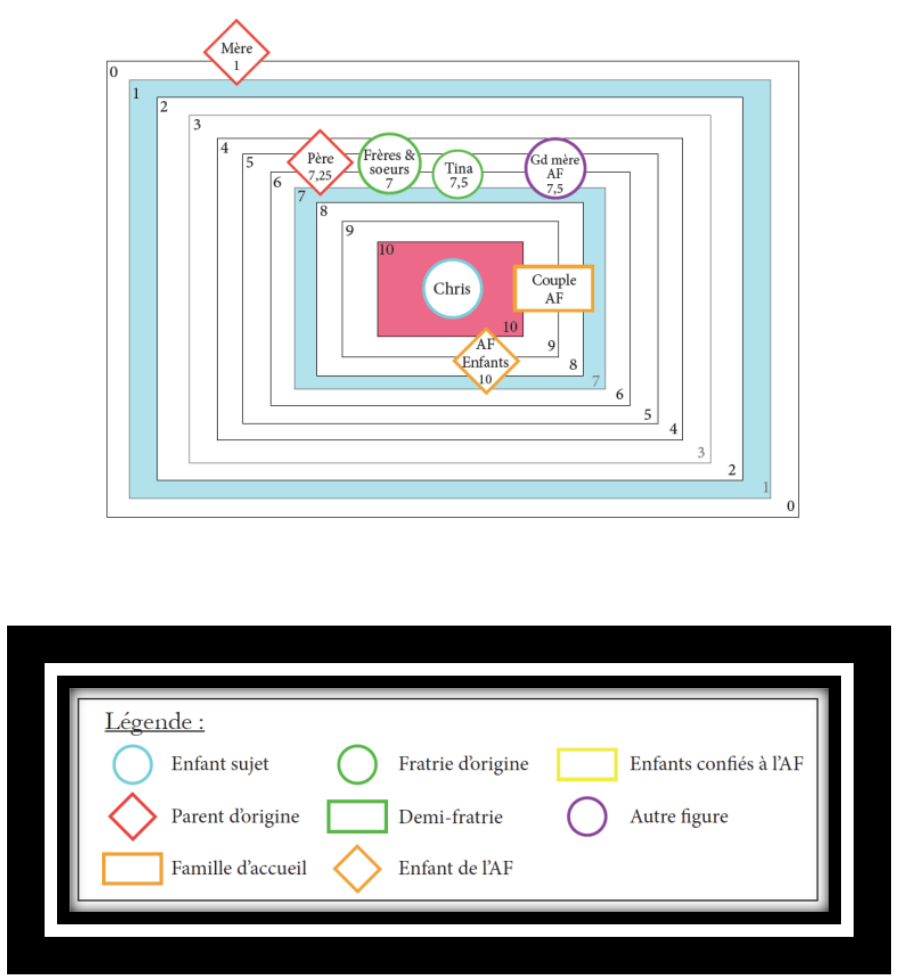

C'est la famille d'accueil qui devient la famille de référence par l'exercice de ses fonctions d'accueil, d'éducation, de soutien et d'amour. Fonctions qui sont posées par les adultes et vécues par les enfants comme des actes structurants pour l'enfant et indispensables à son bon développement, fonctions aussi le plus souvent identifiées comme peu existantes chez les 
parents d'origine par les enfants. Ici, la famille de référence de l'enfant est la famille qui l'éduque, qui lui " apprend à faire des choses », une famille qui accueille l'enfant. "C'est eux qui m'ont appris à faire plein de choses, c'est eux qui m'ont accueillie» (Manuelle). "Nathalia c'est comme une mère, je suis arrivée j'avais 14 mois, elle m'a tout appris, comme faire les lacets, c'est comme une mère, elle a fait le travail qu'une mère fait. " (Chris) Ainsi, les enfants distinguent bien celle qui est biologiquement et juridiquement la mère, et celle qui est «comme si », la mère d'accueil... La définition de la famille donnée par cet enfant est révélatrice de cette distinction.

«La famille... c'est les gens qui t'ont élevé, les personnes qui sont là pour toi au quotidien. Ma famille c'est la famille à Carole... la grand-mère à Carole, ses sœurs, les gens qui m'ont élevé. Mes sæurs aussi, mon père, mon frère mais quand je suis malade, c'est Carole qui est là au quotidien, si je me casse une jambe c'est Carole qui sera là. Alors ma famille c'est la famille à Carole, par contre je sais que j'ai une autre famille, on a la famille de tous les jours et ma famille de sang et de mon nom. "S'il y a reconnaissance de l'existence de deux familles, une seule famille fait référence pour l'enfant, celle de la stabilité du quotidien.

«La famille de temps en temps » c'est la famille d'origine, qui reste présente compte tenu du « lien du sang » qui attache l'enfant à sa parenté. L'enfant voit ponctuellement ses parents, ses frères et sœurs en fonction du rythme des visites. Si les parents sont mentionnés comme constituant une autre famille, celle des «liens du sang», ils sont positionnés bien souvent après la famille d'accueil, et ce quel que soit l'âge des enfants.

\section{$B$ « Mes deux familles »}

Dans cette catégorie, l'enfant ne peut faire de choix entre les deux familles. La dette de vie parentale se retrouve bien scindée en deux, mais sans que la dette à l'égard des parents d'origine, qui ont donné la vie, soit déniée, au contraire d'autres situations. Julie est une enfant de 7 ans, placée depuis l'âge de 4 ans dans sa famille d'accueil avec ses deux demisœurs Amélia (9 ans) et Mélinda (13 ans). La demi-sœur aînée (utérine) Maéva a été placée dans une autre famille d'accueil. Quant aux petits-frères jumeaux âgés de 3 ans, ils sont restés au domicile parental avec les parents d'origine de Julie.

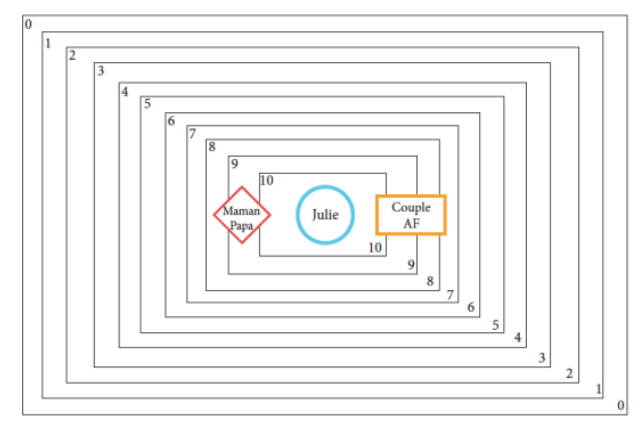

"Je sais ce que c'est une famille - c'est vivre tous ensemble! - mais je ne sais pas répondre à ta question.... parce que je ne sais pas avec quelle famille. Maman ou Tatie ? Parce que j'ai deux familles! Ah-ah-ah maman et tatie! Mais moi je me sens bien ici, mais je voudrais bien que, une fois par semaine, des fois enfin... dimanche par exemple.... je vais voir ma maman et jusqu'à dimanche je vais voir tatie! Voilà! Je voudrais bien que ça se passe comme ça!» 
L'enfant nous décrit ici une configuration familiale que l'on retrouve lors de séparation conjugale et la mise en place d'une garde résidence entre les deux parents, dont la majeure partie du temps est passé chez l'un des parents, et une mineure chez l'autre parent sous la forme de week-end. Au-delà de ce qui est souhaité par l'enfant, c'est aussi la nécessaire reconnaissance de l'importance de chaque famille dans le maintien d'un fragile équilibre pour l'enfant.

\section{$\mathrm{C}$ «Mes parents seulement »}

Seul exemple de cette position, Éric a été placé plus de 10 ans dans sa famille d'accueil, aujourd'hui en bénéficiant d'une aide jeune majeur. Il vit dans une chambre juste à côté de la maison de la famille d'accueil. Encore scolarisé, il passe une partie de son temps chez la famille d'accueil, notamment pour les repas. Alors que nous le pensions fortement attaché à sa famille d'accueil, compte tenu du nombre d'années passées dans cette famille, l'entretien montre que ce n'était pas le cas. Il exprime peu de sentiments à l'égard de cette famille, pour lui c'est simplement une famille qui l'accueille depuis plusieurs années. Il voit son père une fois par mois, et sa mère l'appelle toutes les semaines. Il considère que seuls ses parents font partie de sa famille.

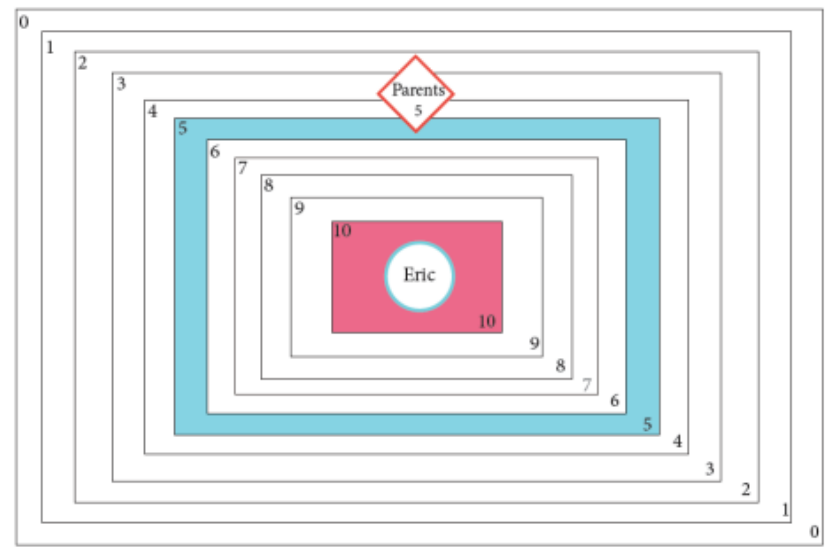

\section{$D$ « Ni mes parents, ni ma famille d'accueil »}

Nous constatons que certains jeunes considèrent ne pouvoir s'appuyer ni sur leur famille d'origine, ni sur leur famille d'accueil. Ces jeunes sont souvent plus âgés, à la marge du service de protection de l'enfance, soit parce qu'ils vont le quitter bientôt, soit parce qu'ils en sont sortis et posent alors un regard rétrospectif sur leur histoire. Ces jeunes se tournent vers une troisième figure familiale liée à une possible alliance, figure familiale du futur conjoint. «La famille de mon copain, c'est comme ma famille, c'est ma famille de cœur, quand je vais chez eux, on dirait que je vais chez moi.... Là-bas je me sens mieux qu'ici.»(Charline). 


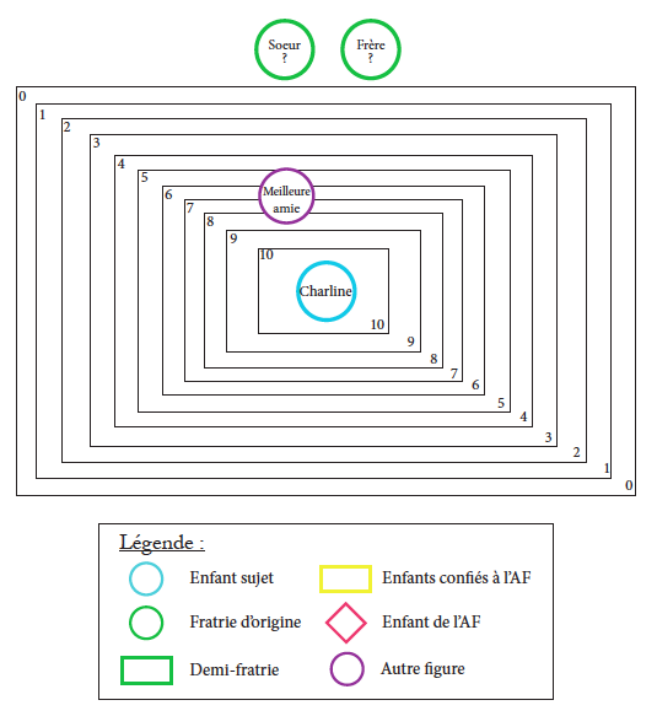

Certains enfants se démarquent des précédentes positions, trouvant ailleurs leur famille, en dehors des références d'origine et d'accueil. Pour eux la famille c'est autre chose, elle ne serait associée ni aux données biologiques, ni à l'éducation reçue dans le cadre de l'accueil, mais aux sentiments, aux ressentis qu'ils éprouvent pour d'autres structures familiales comme leur belle-famille. Ainsi, comme le soulignent Chantal Collard et Françoise Zonabend, l'individu est toujours en quête d'affiliation. Ils n'existent pas d'homme hors parenté. « $L a$ parenté est inscrite dans toutes sociétés, au point qu'on ne peut s'en dispenser même si d'un groupe social à l'autre, d'une époque à l'autre, on observe d'importantes variations». Les auteurs soulignent que si les demandes de compréhension autour de ce qui fait "parenté » n'ont jamais été aussi prégnantes, les mouvements d'acceptation ou de rejets familiaux sont aussi forts ${ }^{27}$.

\section{Discussion}

\section{a. La solidarité familiale, valeur de lien}

L'analyse des quatre formes de famille pour l'enfant confié, montre que la famille d'accueil, «la famille où je vis », est majoritairement la famille de référence pour l'enfant. Cela s'explique avant tout par le temps passé dans la famille, mais aussi par la notion de soutien auquel font référence tous les enfants. Ils attribuent quasiment tous la fonction d'aide à la famille d'accueil, estimant qu'elle fait défaut à leurs parents qui restent dans l'ensemble peu présents. Il va de soi que, pour les enfants, la famille est présente pour le meilleur et pour le pire, et qu'en cas de coup dur, on peut toujours compter sur les siens. Si l'affection est le ciment de la famille, l'expression de solidarités familiales l'est tout autant. Or, on constate que nombre de parents ne sont pas évoqués par les enfants dès lors qu'un problème se présente, mais c'est bien l'assistante familiale et sa famille qui exercent cette fonction de solidarité et de soutien, aussi bien pendant l'enfance qu'à l'entrée à l'âge adulte. Les enfants évoquent alors la relation au parent en faisant le plus souvent référence à ses difficultés, venant apporter une raison explicative du peu, voire du manque de soutien parental. La famille d'accueil est de ce fait le plus souvent celle sur laquelle l'enfant s'appuie. C'est ce que nous dit Mélissa une enfant confiée, "La famille c'est plus une question de avec qui on se sent bien, et sur qui on peut compter en fait!» 
En d'autres termes, le sentiment qui unit, le temps passé ensemble et la sécurité donnée par la capacité reconnue à prendre soin, c'est cela qui fait famille pour les enfants accueillis. On constate une «culture familiale de solidarité ${ }^{28}$, un contrat tacite entre l'enfant accueilli et sa famille d'accueil, inscrits dans le croisement de leurs histoires, mettant en jeu l'absence de réel soutien parental et l'inscription de l'enfant dans la famille d'accueil, avec des pratiques d'échanges au quotidien, de transmission, d'un discours de soutien de la famille d'accueil. L'entraide et l'exercice de la solidarité d'accueil ont valeur de lien. Même si certains parents sont présents par des visites régulières à la maison des solidarités ou à leur domicile, les enfants dans la majorité des cas étudiés s'appuient sur la famille d'accueil en cas de difficulté et non sur leur famille d'origine, identifiant très tôt les possibles comportements familiaux d'entraide ou non, du donner et du recevoir.

\section{b. Des formes de familles ajustées}

Dans sa recherche sur les trajectoires de vie des enfants placés et les liens familiaux, Françoise-Romaine Ouellette demandait aux enfants de représenter par un dessin leur famille ${ }^{29}$. Une majorité des enfants représentaient leur famille d'origine comme étant leur famille. Toutefois, ces résultats diffèrent selon l'âge moyen des enfants au moment du placement. En effet plus l'enfant est jeune, plus il a tendance à représenter sa famille par la famille d'accueil, et inversement, lorsque l'âge est plus élevé, il identifie davantage sa famille à sa famille d'origine. Dans d'autres travaux, il n'a pas été tenu compte de cette distinction par âge $^{30}$. Ils mentionnent une identification massive des jeunes enfants placés à leur famille d'accueil et non à leur famille d'origine. Néanmoins, la recherche de Françoise-Romaine Ouellette permet de penser que si l'affect est le principal critère pour les plus jeunes, avec l'âge la compréhension de la filiation juridiquement instituée se place en concurrence. ${ }^{31}$

La recherche de Sara Boivin ${ }^{32}$ sur les représentations sociales du placement chez des enfants de sept à dix ans en famille d'accueil approfondit la question en montrant que ces enfants font majoritairement référence à leur famille d'accueil comme leur principale famille, avec les couples d'accueil comme personnes les plus significatives dans leur vie. Ils se sentent bien dans leur famille d'accueil et tiennent des propos très positifs à son égard. A l'inverse, lorsque l'enfant a vécu plusieurs années au sein de sa famille biologique, il garde un certain attachement au parent biologique, ainsi qu'à sa fratrie lorsqu'ils vivaient ensemble dans la même famille. Mais, quelle que soit la situation, les enfants rencontrés ont une vision généralement positive d'eux-mêmes et de leur placement. Ils se disent heureux, contents, joyeux de vivre en famille d'accueil.

Les quatre formes de famille identifiées pour les enfants confiés complètent ces différentes recherches. Si le couple d'accueil arrive en première position de ce qui fait famille pour les enfants, ils sont parfois associés à leurs enfants (Nina, Mélodie), ou aux frères et sœurs d'origine de l'enfant confié (Cyndie, Khelia), selon la configuration familiale (présence ou

28 Claudine ATTIAS-DONFUT, Nicole LAPIERRE, Martine SEGALEN, Le nouvel esprit de famille, Odile Jacob, 2002.

29 Françoise-Romaine OUELLETTE, Johanne CHARBONNEAU, Ercillia PALACIO-QUINTIN, Colette JOURDAN-IONESCU, Le placement en famille d'accueil : liens familiaux et dynamiques de réseau, INRS-Urbanisation, Culture et Société, 2001.

30 Sara BOIVIN, Les représentations du placement chez les enfants de sept à dix ans places à long terme, Mémoire, Université Laval, Maîtrise en sciences sociales, Québec, 2009 ; FESTIGER Trudy, No one ever asked us.... A postscript to foster care, Columbia University Press, 1983.

31 Françoise-Romaine OUELLETTE, Johanne CHARBONNEAU, Ercillia PALACIO-QUINTIN, Colette JOURDAN-IONESCU, 2001, op.cit.

32 Sara BOIVIN, 2009, op.cit. 
non de frères et sœurs ou d'enfants de la famille d'accueil) et en fonction de l'histoire de l'enfant. La présence de frères et sœurs d'origine induit généralement des positions nuancées, mais il arrive parfois que certains s'identifient essentiellement à la famille d'accueil, mettant en second plan la fratrie d'origine (Christophe, Lila). D'autres enfants ayant des relations régulières avec leurs parents, ou l'un d'entre eux, illustrent des configurations familiales pluridimensionnelles, mettant les composantes d'accueil et d'origine quasiment au même niveau, tout en donnant la primauté de vie dans le cadre de leur famille d'accueil (Neily, Thierry). Pour les enfants en conflit, placés depuis peu, où en placement tardif à l'adolescence, les configurations familiales sont marquées par l'incertitude, voire la rupture, la famille de référence renvoyant à un ailleurs, ni celle de l'accueil, ni celle d'origine (Sofie). Ce que l'enfant confié considère comme «sa vraie » famille est complexe, autorisant des variations importantes, sans pouvoir opposer un «vrai » et un «faux», les différentes facettes de sa famille venant composer le puzzle de son existence.

On rejoint la position développée par Jacqueline Wendland et Justine Gaugue-Finot concernant le développement du sentiment d'affiliation chez les enfants placés en famille d'accueil. "Lorsqu'un sentiment d'affiliation se construit envers la famille d'accueil, celui-ci n'empêche pas le maintien d'un lien familial avec les parents biologiques. » ${ }^{33}$ Les auteures constatent que certains enfants témoignent d'une double appartenance. Elles proposent de concevoir le placement familial autrement, de reconnaître cette possibilité pour l'enfant de se sentir membre des deux familles, non pas comme une concurrence mais plutôt comme une coopération entre deux familles. Cela rejoint les travaux de recherche en cours sur la parentalité d'accueil en protection de l'enfance, et les modes de suppléance offrant une nouvelle lecture des liens de la filiation d'origine aux affiliations possibles ${ }^{34}$.

\section{Conclusion...Une ouverture vers les parentés plurielles}

La dernière enquête européenne sur les valeurs familiales ${ }^{35}$, montre que pour $87 \%$ des français, la famille est le domaine de la vie qu'ils considèrent comme le plus important, devant le travail, les amis et les loisirs. Réussir sa vie familiale est perçu comme la première condition au bonheur (54\%), loin devant la réussite en amour (24\%) ou dans le champ professionnel $(8 \%)$. La famille est donc une valeur forte à toutes les périodes de la vie, y compris à celles de la jeunesse et du processus d'autonomisation ${ }^{36}$. La famille est considérée comme l'un des derniers espaces de sécurité et de solidarité face à l'adversité. C'est aussi ce que nous disent les enfants confiés en ce qui concernent leur perception de la famille. Une famille est tout d'abord un lieu où l'on vit ensemble, une cellule où l'on se soutient, dont les membres partagent des moments de vie, une partie du quotidien, et de l'amour. En accueil familial, la famille d'accueil exerce une partie importante des fonctions parentales, à la fois éducatives et affectives. Elle permet à l'enfant confié d'établir des liens familiaux nouveaux et inédits, tout en étant enjointe à tenir une position professionnelle. Ainsi la place et les fonctions de la famille d'accueil participent à un processus d'élaboration de lien affectif de proximité émanant d'une position parentale sans en avoir le statut et tout en gardant une

33 Jacqueline WENDLAND, Justine GAUGUE-FINOT, «Le développement du sentiment d'affiliation des enfants placés en famille d'accueil pendant ou après leur petite enfance », Devenir, vol. 4/20, 2008.

34 Nathalie CHAPON, 2014, op.cit.

35 Enquête "Valeurs des européens », Association pour la recherche sur les systèmes de valeurs, 2008.

36 Sébastien DUPONT, «Les familles françaises », Sciences humaines, ${ }^{\circ}$ 292, mai, 2017. 
posture professionnelle. Ces aspects contradictoires, soumettent les familles d'accueil à des injonctions sous tension ${ }^{37}$, et les enfants accueillis à se demander s'ils appartiennent à la famille d'accueil ${ }^{38}$. Ces deux points importants qui constituent le nœud des contradictions affectives et filiatives en accueil familial, ont été étudiés dans de récents travaux en France et en Europe ${ }^{39}$. On se trouve face à un contexte de dissociation des composantes de la parentalité où les modes de transmission et d'échanges sont bouleversés entre ascendants et descendants, questionnant le rapport à la filiation. L'enfant confié vit une histoire familiale singulière composé de différents liens significatifs pour lui, de sentiments d'appartenance, qu'ils soient d'origine ou d'accueil, ouvrant à une possible affiliation.

Le questionnement d'une possible affiliation en accueil familial n'est pas vraiment récent, mais l'évolution à la fois des structures familiales et l'émergence des droits de l'enfant l'a rendu plus accru. En effet, depuis des années, Myriam David a évoqué tout au long de ses écrits la question difficile et omniprésente du sentiment d'appartenance de l'enfant confié, interrogeant ainsi la notion d'affiliation, et la possibilité d'appartenir à plusieurs familles tout en laissant aux parents une place de choix ${ }^{40}$. Aujourd'hui pour de nombreux auteurs faire partie d'une famille pour un enfant confié ne dépendrait plus d'une réalité biologique ou quotidienne, mais plutôt d'une réalité symbolique du sujet ${ }^{41}$. Ainsi certains auteurs comme Paul D. Steinhauer ${ }^{42}$, Catherine Sellenet ${ }^{43}$ ou Salomon ${ }^{44}$, mais aussi Adeline Gouttenoire ${ }^{45}$, évoquent la possibilité pour les parents de l'enfant confié à long terme d'exercer une parentalité partielle ou double parentalité, qui pourrait permettre à l'enfant de s'attacher à sa famille d'accueil sans pour autant effacer ses parents biologiques et sans être pris dans un conflit de loyauté. La dernière recherche que nous avons réalisée sur les liens en famille

37 Catherine SELLENET, «L'accueil familial et ses paradoxes », in CHAPON Nathalie (dir.), Parentalité d'accueil et Mémoire, Pup, Aix-en-Provence, 2016.

38 David REIMER, «Articulation des politiques publiques et des intérêts de l'enfant », Conférence plénière, Journée d'études «Entre continuités et ruptures dans l'accueil familial », Université ParisNanterre/ONPE, 29 septembre 2017.

39 Se référer aux différents travaux présentés lors de la journée d'études de Université ParisNanterre/ONPE, Journée d'étude "Entre continuités et ruptures dans l'accueil familial », Université Paris-Nanterre, 29 septembre 2017 ; Nathalie CHAPON, Sylvio PREMOLI, La parentalité d'accueil en Europe : regards théoriques et pratiques professionnelles, Pup, Aix-en-Provence, 2018.

40 Myriam DAVID, Le placement familial : de la pratique à la théorie, ESF, 1989.

41 Gérard NEYRAND, «La parentalité d'accueil», in Nathalie CHAPON, Gérard NEYRAND, Dialogue : Parentalité et famille d'accueil, $\mathrm{n}^{\circ}$ 167, 2005 ; Catherine SELLENET, «Vivre en deux familles. Quels repères familiaux pour les enfants élevés en famille d'accueil », Communication XVème congrès AIFREF, 2013 ; Daniel COUM, Avons-nous besoin de père et de mère, Erès, 2016 ; Jacqueline WENDLAND, Justine GAUGUE-FINOT, op .cit.

42Paul D. STEINHAUER, Le moindre mal. La question du placement de l'enfant, Presses universitaires de Montréal, 1996.

43 Catherine SELLENET, La parentalité décryptée. Pertinences et dérives d'un concept, L'Harmattan, 2007.

44 O. SALOMON, «Avec les parents, de quoi se mêle-t-on exactement ? » in D. BASS, A. PELLÉ (dir.), L'art d'accompagner en placement familial. Du salon du prêt-à-penser à l'artisanat du surmesure, Erès, 1999, pp. 187-196.

45 Adeline GOUTTENOIRE, «Quarante propositions pour adapter la protection de l'enfance et l'adoption aux réalités d'aujourd'hui », Droit famille, $\mathrm{n}^{\circ}$ 9, sept. 2014, dossier 5; Adeline GOUTTENOIRE, Frédérique EUDIER, «Une réforme impressionniste », JCP G, n 16, 2016, p. 479. 
d'accueil ${ }^{46}$ croise les différentes approches sociologiques et juridiques, et propose de nouvelles orientations juridiques face aux réalités familiales d'accueil. Au-delà, c'est aussi l'évolution du modèle de pensée en protection de l'enfance qui est questionnée. Ce modèle de pensée a glissé d'un courant tourné vers l'éviction des parents à une posture plutôt opposée, la protection de la famille et des parents. Cette conception pro-familiale a aujourd'hui montré ses limites, puisque de nombreux enfants restent confiés toute leur enfance dans un système qui n'est pas sécurisé et pas assez protecteur pour eux. Assurer la continuité de la prise en charge des enfants en famille d'accueil constitue de plus en plus un objectif pour les systèmes de protection de l'enfance, aussi bien en France qu'en Europe ${ }^{47}$. Si pendant des millénaires la famille a dû répondre à un système de filiation avec des normes précises imposant un ordre social et familial des enfants à naître, pour Maurice Godelier il est aujourd'hui devenu possible d'élargir le système de parenté à l'amour et au désir des individus. Cette position inédite ouvrant le champ de la parenté aux affiliations possibles permettrait ainsi aux enfants confiés de vivre leur pluralité de lien en toute sécurité et sérénité, même si le reconnait Maurice Godelier cela entraînera "plus de responsabilités que jamais pour les adultes vis-àvis des enfants. Car finalement, on en revient toujours à l'enfant: sans lui, rien de ce que nous avons inventé n'aurait continué d'exister» ${ }^{48}$.

46 Nathalie CHAPON, Gérard NEYRAND, Caroline SIFFREIN-BLANC, Les liens en famille d'accueil, Erès, 2018.

47 Journée d'études de Université Paris-Nanterre/ONPE, Journée d'étude "Entre continuités et ruptures dans l'accueil familial », Université Paris-Nanterre, Table ronde : "Continuités et ruptures dans la pluralité des liens ».

48 Maurice GODELIER, «Systèmes de parenté et formes de famille», Recherches de sciences religieuses, 3, t. 2, 2014. 